\title{
Trajectory Generation of Biped Running Robot with Minimum Energy Consumption
}

\author{
Yasutaka Fujimoto \\ Department of Electrical and Computer Engineering \\ Yokohama National University \\ Yokohama 240-8501 Japan \\ Email: fujimoto@ynu.ac.jp
}

\begin{abstract}
The exact and general formulation of optimal control for biped robots based on numerical representation of motion equation is proposed. We can solve exactly the minimum energy consumption trajectories for a biped running motion. Through the numerical study of a five link planar biped robot, it is found that big peak power and torque is required for the knee joints but its consumption power is small and the main work is done by the hip joints.
\end{abstract}

\section{INTRODUCTION}

Recent years, many studies have reported analysis of bipedal locomotion and its application to bipedal robots[1]-[10]. Several autonomous humanoid robots are actually developed[4][8]. Generally, careful design is required for development of a bipedal robot. Selection of gears and actuators, taking their rate, power, torque, and weight into account, is especially important. Development of rare-earth permanent magnet materials Nd-Fe-B and power conversion technology based on semiconductor switching devices in the last decade enables improvement of power/weight ratio of actuators and realization of such autonomous bipedal robots. However, they are still underpowered to achieve fast walking and running motions. There are several researches on running control of bipedal model[11]-[13]. Kajita, et. al. reported that their proposed running pattern requires at least 28 to 56 times more powerful actuators than that of their actual humanoid robot HRP1, and also the consumption power is estimated ten times bigger than the human runner.

It is useful to know the lower bound of the consumption energy when we design the bipedal robot and select actuators. In this paper, a method to generate a trajectory of a running motion with minimum energy consumption is proposed. The generation of low-energy trajectories for biped robots remained an open problem. Usually, it is formulated as an optimal control problem. Since symbolic expression of motion equation of robots becomes extremely complicated in the case that the number of links increases, only specific simple type of a structure of robots was investigated and simplified assumptions such as ignoring the effects of centripetal forces were made in the past works[3]. In this paper, exact and general formulation of optimal control for biped robots based on numerical representation of motion equation is proposed to solve exactly the minimum energy consumption trajectories.

The rest of the paper is organized as follows. In Section II, the problem definition is introduced where the formulation of the biped running robot is given. The minimization of consumption energy is explained in the Section III, and the computational scheme is proposed in the Section IV. The numerical study of a five link planar biped robot is provided in Section V, and conclusions are outlined in the Section VI.

\section{Model of Biped RunNing Robot}

Consider a three dimensional bipedal robot with open-chain mechanism consisting of $N$ joints and $N+1$ rigid links. Since the robot is not fixed on the ground, it is modeled as a free-fall manipulator which has $N+6$ motion-degree-offreedom[14][15]. The model in general form is given by

$$
\boldsymbol{H} \ddot{\boldsymbol{x}}+\boldsymbol{C} \dot{\boldsymbol{x}}+\boldsymbol{g}=\boldsymbol{u}
$$

where $\boldsymbol{H} \in \mathcal{R}^{(N+6) \times(N+6)}$ is an inertia matrix, $\boldsymbol{C} \in$ $\mathcal{R}^{(N+6) \times(N+6)}$ specifies centrifugal and Coriolis effects, $\boldsymbol{g} \in$ $\mathcal{R}^{N+6}$ specifies gravity effects, $\boldsymbol{x}=[\boldsymbol{\theta}, \boldsymbol{\varphi}, \boldsymbol{p}]^{\top} \in \mathcal{R}^{(N+6)}$ specifies displacements of joints, and posture and position of a base link. $\boldsymbol{u}=[\boldsymbol{n}, \mathbf{0}, \mathbf{0}]^{\top} \in \mathcal{R}^{(N+6)}$ specifies input generalized forces. Also $\boldsymbol{\theta}, \boldsymbol{n} \in \mathcal{R}^{N}$ specify joint angles and input joint torques, respectively. $\varphi, \boldsymbol{p} \in \mathcal{R}^{3}$ specify posture and position of a base link, respectively. An example of the coordinates for the case of planner biped is shown in Fig. 1.

The motion of running is decomposed to two phases; singleleg support phase and flight phase.

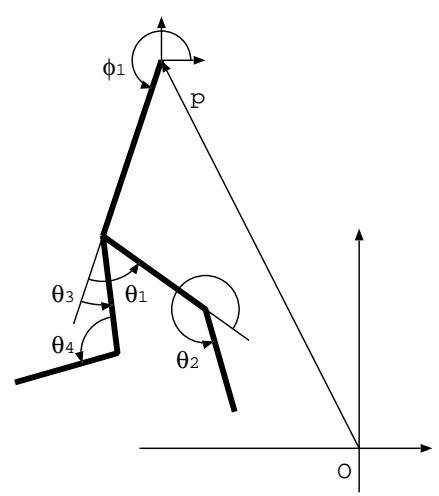

Fig. 1. A five link biped robot. 


\section{A. Model of Support Phase}

Let the tip position of the support leg with respect to the origin of the base link system be $\boldsymbol{h}(\boldsymbol{\theta}, \boldsymbol{\varphi})$. Then its position with respect to the origin of the world coordinate system is represented by

$$
\boldsymbol{y}=\boldsymbol{h}(\boldsymbol{\theta}, \varphi)+\boldsymbol{p} .
$$

Since the foot of the support leg is fixed on the ground during single support phase, it is subject to the following conditions.

$$
\begin{aligned}
& \dot{y}=\boldsymbol{J} \dot{\theta}+\boldsymbol{R} \dot{\varphi}+\dot{p}=\mathbf{0} \\
& \ddot{y}=\boldsymbol{J} \ddot{\theta}+\dot{J} \dot{\theta}+R \ddot{\varphi}+\dot{R} \dot{\varphi}+\ddot{p}=\mathbf{0}
\end{aligned}
$$

where $\boldsymbol{J}=\partial \boldsymbol{h} / \partial \boldsymbol{\theta}^{\top}$ and $\boldsymbol{R}=\partial \boldsymbol{h} / \partial \boldsymbol{\varphi}^{\top}$ are Jacobian matrices.

Eliminating $\dot{p}$ and $\ddot{p}$ from (1) using (3) and (4), the following dynamics is obtained.

$$
\boldsymbol{H}_{s} \ddot{\boldsymbol{x}}_{s}+\boldsymbol{C}_{s} \dot{\boldsymbol{x}}_{s}+\boldsymbol{g}_{s}=\boldsymbol{u}_{s}
$$

where $\boldsymbol{x}_{s}=\left[\boldsymbol{\theta}_{s}, \boldsymbol{\varphi}_{s}\right]^{\top}$ and $\boldsymbol{u}_{s}=\left[\boldsymbol{n}_{s}, \mathbf{0}\right]^{\top}$. The subscript $s$ represents variables during support phase. The state equation is given by

$$
\dot{\boldsymbol{w}}_{s}=\left[\begin{array}{c}
\dot{\boldsymbol{x}}_{s} \\
\boldsymbol{H}_{s}^{-1}\left(\boldsymbol{u}_{s}-\boldsymbol{C}_{s} \dot{\boldsymbol{x}}_{s}-\boldsymbol{g}_{s}\right)
\end{array}\right]
$$

where $\boldsymbol{w}_{s}=\left[\boldsymbol{x}_{s}, \dot{\boldsymbol{x}}_{s}\right]^{\top}=\left[\boldsymbol{\theta}_{s}, \boldsymbol{\varphi}_{s}, \dot{\boldsymbol{\theta}}_{s}, \dot{\boldsymbol{\varphi}}_{s}\right]^{\top} \in \mathcal{R}^{2 N+6}$ is a state vector.

\section{B. Model of Flight Phase}

During flight phase, there is no restriction on the motion equation (1). The conservation law of angular momentum is already included equivalently in (1), which corresponds to no existence of external forces. Therefore the dynamics becomes

$$
\boldsymbol{H}_{f} \ddot{\boldsymbol{x}}_{f}+\boldsymbol{C}_{f} \dot{\boldsymbol{x}}_{f}+\boldsymbol{g}_{f}=\boldsymbol{u}_{f}
$$

where $\boldsymbol{x}_{f}=\left[\boldsymbol{\theta}_{f}, \boldsymbol{\varphi}_{f}, \boldsymbol{p}_{f}\right]^{\top}$ and $\boldsymbol{u}_{f}=\left[\boldsymbol{n}_{f}, \mathbf{0}, \mathbf{0}\right]^{\top}$. The subscript $f$ represents variables during flight phase. The state equation is given by

$$
\dot{\boldsymbol{w}}_{f}=\left[\begin{array}{c}
\dot{\boldsymbol{x}}_{f} \\
\boldsymbol{H}_{f}^{-1}\left(\boldsymbol{u}_{f}-\boldsymbol{C}_{f} \dot{\boldsymbol{x}}_{f}-\boldsymbol{g}_{f}\right)
\end{array}\right]
$$

where $\boldsymbol{w}_{f}=\left[\boldsymbol{x}_{f}, \dot{\boldsymbol{x}}_{f}\right]^{\top}=\left[\boldsymbol{\theta}_{f}, \boldsymbol{\varphi}_{f}, \boldsymbol{p}_{f}, \dot{\boldsymbol{\theta}}_{f}, \dot{\boldsymbol{\varphi}}_{f}, \dot{\boldsymbol{p}}_{f}\right]^{\top} \in$ $\mathcal{R}^{2(N+6)}$ is a state vector.

\section{Minimum Energy Consumption Gait}

It is useful to know the lower bound of the consumption energy when we design the bipedal robot and select actuators. An ideal actuator is assumed in this paper to investigate the consumption energy, although real actuators with highratio gears such as harmonic gears have large frictions and roughly $70 \%$ of energy efficiency. The energy regeneration is considered. The problem is to find input joint torques and initial posture that minimize input energy during running motion under the condition that the robot takes completely periodic and symmetric motion, given the step period and the stride. The problem is described as follow.

$$
\begin{array}{cl}
\text { minimize } & E=\int_{0}^{T} \dot{\boldsymbol{\theta}}^{\top} \boldsymbol{n} d t \\
\text { subject to } & \boldsymbol{\theta}(T)=\boldsymbol{K} \boldsymbol{\theta}(0) \\
& \boldsymbol{\varphi}(T)=\boldsymbol{\varphi}(0) \\
& \boldsymbol{p}(T)=\boldsymbol{p}(0)+\boldsymbol{S}
\end{array}
$$

where $T$ is a period for one step. $|\boldsymbol{S}|$ is the stride. $K$ is a coordinate conversion matrix;

$$
\boldsymbol{K}=\left[\begin{array}{ccc}
\boldsymbol{I}_{b} & 0 & 0 \\
0 & 0 & \boldsymbol{I}_{\ell} \\
0 & \boldsymbol{I}_{\ell} & 0
\end{array}\right]
$$

where $\boldsymbol{I}_{b}$ and $\boldsymbol{I}_{\ell}$ are identity matrices whose dimensions are same as number of joints in body and one leg, respectively.

Since the structure of dynamics is variable depending on the phase as shown in the previous section, a reflection of time axis is introduced. A new time axis is given by

$$
\tau=\left\{\begin{array}{lll}
\frac{t}{\alpha T} & \text { for } \quad 0 \leq t \leq \alpha T \\
\frac{T-t}{(1-\alpha) T} & \text { for } \quad \alpha T \leq t \leq T
\end{array} .\right.
$$

The timing chart of events are shown in Fig. 2. All variables in the rest of this section are functions of $\tau$.

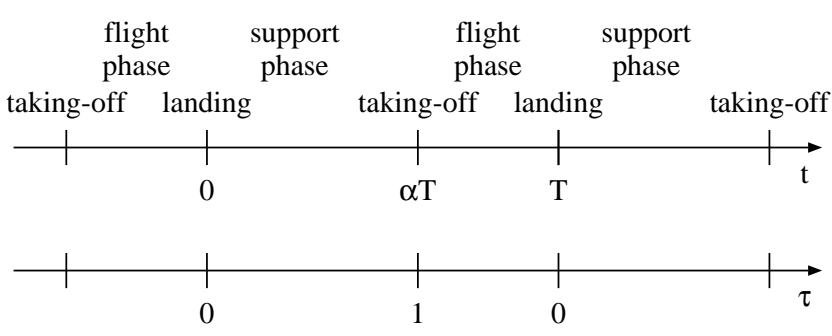

Fig. 2. Timing chart of events.

The objective function (9) is represented by

$$
\begin{aligned}
E & =\int_{0}^{\alpha T} \dot{\boldsymbol{\theta}}_{s}^{\top} \boldsymbol{n}_{s} d t+\int_{\alpha T}^{T} \dot{\boldsymbol{\theta}}_{f}^{\top} \boldsymbol{n}_{f} d t \\
& =\int_{0}^{1}\left(\alpha \dot{\boldsymbol{\theta}}_{s}^{\top} \boldsymbol{n}_{s}+(1-\alpha) \dot{\boldsymbol{\theta}}_{f}^{\top} \boldsymbol{n}_{f}\right) T d \tau .
\end{aligned}
$$

The state equations (6) for $0 \leq t \leq \alpha T$ and (8) for $\alpha T \leq t \leq$ $T$ are transformed onto $\tau$-axis as follows.

$$
\begin{aligned}
\frac{d \boldsymbol{w}_{s}}{d \tau} & =\alpha T\left[\begin{array}{c}
\dot{\boldsymbol{x}}_{s} \\
\boldsymbol{H}_{s}^{-1}\left(\boldsymbol{u}_{s}-\boldsymbol{C}_{s} \dot{\boldsymbol{x}}_{s}-\boldsymbol{g}_{s}\right)
\end{array}\right] \\
\frac{d \boldsymbol{w}_{f}}{d \tau} & =(\alpha-1) T\left[\begin{array}{c}
\dot{\boldsymbol{x}}_{f} \\
\boldsymbol{H}_{f}^{-1}\left(\boldsymbol{u}_{f}-\boldsymbol{C}_{f} \dot{\boldsymbol{x}}_{f}-\boldsymbol{g}_{f}\right)
\end{array}\right]
\end{aligned}
$$

State variables should include the support phase ratio $\alpha$ in order to find its optimal value as well. Then, the following differential equation is introduced.

$$
\frac{d \alpha}{d \tau}=0
$$


Finally, the problem is transformed into a Bolza problem.

$$
\begin{aligned}
& \text { minimize } \quad \widetilde{E}=g\left(\boldsymbol{z}_{1}\right)+\int_{0}^{1}\left(f_{0}(\boldsymbol{z}(\tau), \boldsymbol{v}(\tau))\right. \\
& \left.+\boldsymbol{\lambda}(\tau)^{\top}\left(\frac{d \boldsymbol{z}(\tau)}{d \tau}-\boldsymbol{f}(\boldsymbol{z}(\tau), \boldsymbol{v}(\tau))\right)\right) d \tau
\end{aligned}
$$

where $\boldsymbol{z}=\left[\boldsymbol{w}_{s}, \boldsymbol{w}_{f}, \alpha\right]^{\top}=\left[\boldsymbol{x}_{s}, \dot{\boldsymbol{x}}_{s}, \boldsymbol{x}_{f}, \dot{\boldsymbol{x}}_{f}, \alpha\right]^{\top}=\left[\boldsymbol{\theta}_{s}, \boldsymbol{\varphi}_{s}\right.$, $\left.\dot{\boldsymbol{\theta}}_{s}, \dot{\boldsymbol{\varphi}}_{s}, \boldsymbol{\theta}_{f}, \boldsymbol{\varphi}_{f}, \boldsymbol{p}_{f}, \dot{\boldsymbol{\theta}}_{f}, \dot{\boldsymbol{\varphi}}_{f}, \dot{\boldsymbol{p}}_{f}, \alpha\right]^{\top} \in \mathcal{R}^{4 N+18}$ is a state vector, $\boldsymbol{v}=\left[\boldsymbol{n}_{s}, \boldsymbol{n}_{f}\right]^{\top} \in \mathcal{R}^{2 N}$ is a input vector, $\boldsymbol{\lambda} \in \mathcal{R}^{4 N+12}$ is a Lagrange multiplier, $\boldsymbol{z}_{1}=\boldsymbol{z}(1)$ is state at the terminal period, and

$$
\begin{aligned}
f_{0}(\boldsymbol{z}, \boldsymbol{v})= & \left(\alpha \dot{\boldsymbol{\theta}}_{s}^{\top} \boldsymbol{n}_{s}+(1-\alpha) \dot{\boldsymbol{\theta}}_{f}^{\top} \boldsymbol{n}_{f}\right) T \\
\boldsymbol{f}(\boldsymbol{z}, \boldsymbol{v})= & {\left[\begin{array}{c}
\alpha T \dot{\boldsymbol{x}}_{s} \\
\alpha T \boldsymbol{H}_{s}^{-1}\left(\boldsymbol{u}_{s}-\boldsymbol{C}_{s} \dot{\boldsymbol{x}}_{s}-\boldsymbol{g}_{s}\right) \\
(\alpha-1) T \dot{\boldsymbol{x}}_{f} \\
(\alpha-1) T \boldsymbol{H}_{f}^{-1}\left(\boldsymbol{u}_{f}-\boldsymbol{C}_{f} \dot{\boldsymbol{x}}_{f}-\boldsymbol{g}_{f}\right) \\
0
\end{array}\right] }
\end{aligned}
$$

The function $g\left(\boldsymbol{z}_{1}\right)$ represents penalty for the terminal condition which is introduced to guarantee continuity of the state variable at the instant of the taking-off.

$$
\begin{aligned}
g\left(\boldsymbol{z}_{1}\right)= & W\left(\left\|\boldsymbol{\theta}_{s 1}-\boldsymbol{\theta}_{f_{1}}\right\|^{2}+\left\|\dot{\boldsymbol{\theta}}_{s 1}-\dot{\boldsymbol{\theta}_{f_{1}}}\right\|^{2}\right. \\
& +\left\|\boldsymbol{\varphi}_{s 1}-\boldsymbol{\varphi}_{f_{1}}\right\|^{2}+\left\|\dot{\boldsymbol{\varphi}}_{s 1}-\dot{\boldsymbol{\varphi}}_{f_{1}}\right\|^{2} \\
& \left.+\left\|\boldsymbol{p}_{s 1}-\boldsymbol{p}_{f_{1}}\right\|^{2}+\left\|\dot{\boldsymbol{p}}_{s 1}-\dot{\boldsymbol{p}}_{f_{1}}\right\|^{2}\right)
\end{aligned}
$$

where $W$ is an weight coefficient. The subscript 1 is at $\tau=1$. The variables $\boldsymbol{p}_{s}$, and $\dot{\boldsymbol{p}}_{s}$ are implicitly defined here. They are represented by functions of $w_{s}$.

Variation of the extended objective function $\widetilde{E}$ is given by

$$
\begin{aligned}
\delta \widetilde{E}= & -\boldsymbol{\lambda}_{0}^{\top} \frac{\partial \boldsymbol{z}_{0}}{\partial \boldsymbol{z}_{0}^{\prime \top}} \delta \boldsymbol{z}_{0}^{\prime}+\left(\frac{\partial g}{\partial \boldsymbol{z}_{1}}+\boldsymbol{\lambda}_{1}\right)^{\top} \delta \boldsymbol{z}_{1} \\
& +\int_{0}^{1}\left(\left(\frac{\partial f_{0}}{\partial \boldsymbol{z}}-\frac{\partial f^{\top}}{\partial \boldsymbol{z}} \boldsymbol{\lambda}-\frac{d \boldsymbol{\lambda}}{d \tau}\right)^{\top} \delta \boldsymbol{z}\right. \\
& \left.+\left(\frac{\partial f_{0}}{\partial \boldsymbol{v}}-\frac{\partial f^{\top}}{\partial \boldsymbol{v}} \boldsymbol{\lambda}\right)^{\top} \delta \boldsymbol{v}+\left(\frac{d \boldsymbol{z}}{d \tau}-\boldsymbol{f}\right)^{\top} \delta \boldsymbol{\lambda}\right) d \tau .
\end{aligned}
$$

In this equation, we assume that the initial state $z_{0}$ is a function of certain variables which consist of partial set of the state, namely, a part of the initial state is independent and the other depends on it. Let the independent initial state variables be $\boldsymbol{z}_{0}^{\prime}=\left[\boldsymbol{\theta}_{s}(0), \boldsymbol{\varphi}_{s}(0), \dot{\boldsymbol{\theta}}_{f}(0), \dot{\boldsymbol{\varphi}}_{f}(0), \dot{\boldsymbol{p}}_{f}(0), \alpha(0)\right]^{\top}$. The rest of the initial state are decided by

$$
\begin{aligned}
& \boldsymbol{\theta}_{f}(0)=\boldsymbol{K} \boldsymbol{\theta}_{s}(0) \\
& \boldsymbol{\varphi}_{f}(0)=\boldsymbol{\varphi}_{s}(0) \\
& \boldsymbol{p}_{f}(0)=-\boldsymbol{h}\left(\boldsymbol{\theta}_{s}(0), \boldsymbol{\varphi}_{s}(0)\right)+\boldsymbol{S} \\
& {\left[\begin{array}{c}
\dot{\boldsymbol{\theta}}_{s}(0) \\
\dot{\boldsymbol{\varphi}}_{s}(0)
\end{array}\right] }=\left[\begin{array}{ccc}
\boldsymbol{K} & 0 & 0 \\
0 & \boldsymbol{I} & 0
\end{array}\right] \\
& \times\left(\boldsymbol{I}-\boldsymbol{H}_{f}^{-1} \widetilde{\boldsymbol{J}}_{f}^{\top}\left(\widetilde{\boldsymbol{J}}_{f} \boldsymbol{H}_{f}^{-1} \widetilde{\boldsymbol{J}}_{f}^{\top}\right)^{-1} \widetilde{\boldsymbol{J}}_{f}\right)\left[\begin{array}{l}
\dot{\boldsymbol{\theta}}_{f}(0) \\
\dot{\boldsymbol{\varphi}}_{f}(0) \\
\dot{\boldsymbol{p}}_{f}(0)
\end{array}\right] .
\end{aligned}
$$

The first three equations are coordinate conversion at the instant of landing and the last is the condition of perfectly inelastic collision at the instant of landing. (See Appendix.)

From (23), the following conditions are obtained.

$$
\begin{aligned}
\boldsymbol{\lambda}_{1} & =-\frac{\partial g}{\partial \boldsymbol{z}_{1}} \\
\frac{d \boldsymbol{\lambda}}{d \tau} & =\frac{\partial f_{0}}{\partial \boldsymbol{z}}-\frac{\partial \boldsymbol{f}^{\top}}{\partial \boldsymbol{z}} \boldsymbol{\lambda} \\
\frac{d \boldsymbol{z}}{d \tau} & =\boldsymbol{f}
\end{aligned}
$$

Also the gradients are given by

$$
\begin{aligned}
\frac{\partial \widetilde{E}}{\partial \boldsymbol{z}_{0}^{\prime}} & =-\frac{\partial \boldsymbol{z}_{0}^{\top}}{\partial \boldsymbol{z}_{0}^{\prime}} \boldsymbol{\lambda}_{0} \\
\frac{\partial \widetilde{E}}{\partial \boldsymbol{v}} & =\frac{\partial f_{0}}{\partial \boldsymbol{v}}-\frac{\partial \boldsymbol{f}^{\top}}{\partial \boldsymbol{v}} \boldsymbol{\lambda} .
\end{aligned}
$$

To find the optimal solution, the conjugate gradient method in infinite dimensional space (Hilbert space) is applied to this problem. The procedures of the algorithm are as follows.

1) The initial solution $\left[\boldsymbol{z}_{0}^{\prime}, \boldsymbol{v}(\tau)\right]$ is given.

2) The initial state $z_{0}$ is computed by (24)-(27).

3) The differential equation (30) is solved using $\boldsymbol{z}_{0}$.

4) $\lambda_{1}$ is computed by (28) using the final value $z_{1}$.

5) The differential equation (29) is backwardly solved using $\boldsymbol{\lambda}_{1}$.

6) The gradients for $\boldsymbol{z}_{0}$ and $\boldsymbol{v}(\tau)$ are computed by (31), (32) using $\boldsymbol{z}(\tau), \boldsymbol{\lambda}(\tau)$, and $\boldsymbol{v}(\tau)$.

7) The temporary solution $\left[\boldsymbol{z}_{0}^{\prime}, \boldsymbol{v}(\tau)\right]$ is updated toward the direction of the conjugate gradient.

8) If the gradient is not small enough, return to 2.

Finally, the input joint torques $\boldsymbol{n}(t)$, the joint angles $\boldsymbol{\theta}(t)$, the posture and position (of the base link) $\varphi(t), \boldsymbol{p}(t)$, their derivatives $\dot{\boldsymbol{\theta}}(t), \dot{\boldsymbol{\varphi}}(t), \dot{\boldsymbol{p}}(t)$, and the support phase ratio $\alpha$ are obtained.

A general method to compute the partial derivatives in (24)(32) is proposed in the next section.

\section{Computational Scheme for Partial Derivative}

It is difficult to calculate the partial derivatives in (24)-(32) symbolically, because basically it costs very much to obtain a symbolic expression of the equation of motion (1). In this section, a computational scheme for the partial derivatives based on numerical representation of motion equation is proposed.

Each partial derivative is represented as follows.

$$
\begin{aligned}
& \frac{\partial \boldsymbol{f}}{\partial \boldsymbol{z}^{\top}}=T \times \\
& {\left[\begin{array}{ccccc}
\mathbf{0} & \alpha \boldsymbol{I} & \mathbf{0} & \mathbf{0} & \dot{\boldsymbol{x}}_{s} \\
\alpha \frac{\partial \boldsymbol{f}_{s}}{\partial \boldsymbol{x}_{s}^{\top}} & \alpha \frac{\partial \boldsymbol{f}_{s}}{\partial \dot{\boldsymbol{x}}_{s}^{\top}} & \mathbf{0} & \mathbf{0} & \boldsymbol{f}_{s} \\
\mathbf{0} & \mathbf{0} & \mathbf{0} & (\alpha-1) \boldsymbol{I} & \dot{\boldsymbol{x}}_{f} \\
\mathbf{0} & \mathbf{0} & (\alpha-1) \frac{\partial \boldsymbol{f}_{f}}{\partial \boldsymbol{x}_{f}^{f}} & (\alpha-1) \frac{\partial \boldsymbol{f}_{f}}{\partial \dot{\boldsymbol{x}}_{f}^{\dagger}} & \boldsymbol{f}_{f} \\
\mathbf{0} & \mathbf{0} & \mathbf{0} & \mathbf{0} & 0
\end{array}\right]}
\end{aligned}
$$


where

$$
\begin{aligned}
\boldsymbol{f}_{s} & =\boldsymbol{H}_{s}^{-1}\left(\boldsymbol{u}_{s}-\boldsymbol{C}_{s} \dot{\boldsymbol{x}}_{s}-\boldsymbol{g}_{s}\right) \\
\boldsymbol{f}_{f} & =\boldsymbol{H}_{f}^{-1}\left(\boldsymbol{u}_{f}-\boldsymbol{C}_{f} \dot{\boldsymbol{x}}_{f}-\boldsymbol{g}_{f}\right) .
\end{aligned}
$$

And then,

$$
\frac{\partial f}{\partial \boldsymbol{v}^{\top}}=\left[\begin{array}{cc}
\mathbf{0} & \mathbf{0} \\
\alpha T \boldsymbol{H}_{s}^{-1} \boldsymbol{P}_{s} & \mathbf{0} \\
\mathbf{0} & \mathbf{0} \\
\mathbf{0} & (\alpha-1) T \boldsymbol{H}_{f}^{-1} \boldsymbol{P}_{f} \\
\mathbf{0} & \mathbf{0}
\end{array}\right]
$$

where $\boldsymbol{P}_{s}=[\boldsymbol{I}, \mathbf{0}]^{\top} \in \mathcal{R}^{(N+3) \times N}, \boldsymbol{P}_{f}=[\boldsymbol{I}, \mathbf{0}, \mathbf{0}]^{\top} \in$ $\mathcal{R}^{(N+6) \times N}$ are selection matrices. And also,

$$
\begin{gathered}
\frac{\partial f_{0}}{\partial \boldsymbol{z}}=\left[\begin{array}{c}
\mathbf{0} \\
\mathbf{0} \\
\alpha T \boldsymbol{n}_{s} \\
\mathbf{0} \\
\mathbf{0} \\
\mathbf{0} \\
\mathbf{0} \\
(1-\alpha) T \boldsymbol{n}_{f} \\
\mathbf{0} \\
\mathbf{0} \\
T\left(\dot{\boldsymbol{\theta}}_{s}^{\top} \boldsymbol{n}_{s}-\dot{\boldsymbol{\theta}}_{f}^{\top} \boldsymbol{n}_{f}\right)
\end{array}\right] \\
\frac{\partial f_{0}}{\partial \boldsymbol{v}}=\left[\begin{array}{c}
\alpha T \dot{\boldsymbol{\theta}}_{s} \\
(1-\alpha) T \dot{\boldsymbol{\theta}}_{f}
\end{array}\right]
\end{gathered}
$$

$$
\frac{\partial g}{\partial \boldsymbol{z}_{1}}=2 W \times
$$

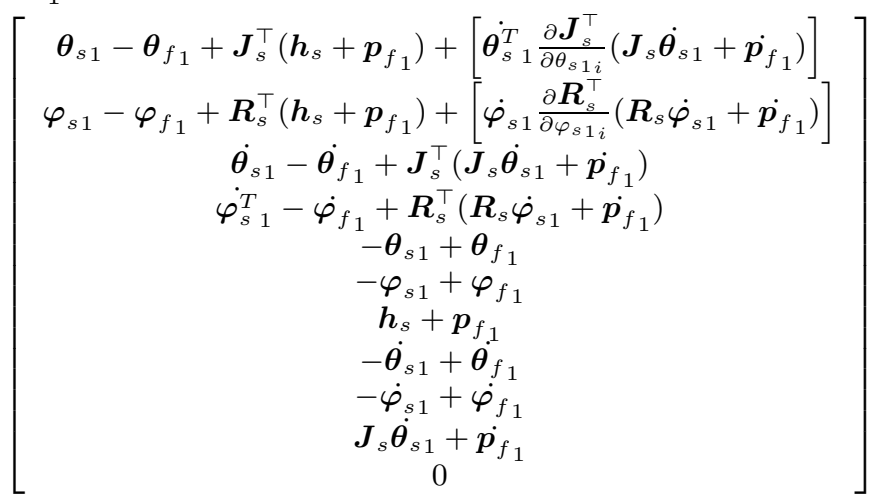

$$
\frac{\partial \boldsymbol{z}_{0}}{\partial \boldsymbol{z}_{0}^{\prime \top}}=\left[\begin{array}{ccc}
\boldsymbol{I} & \mathbf{0} & \mathbf{0} \\
\boldsymbol{\Gamma} & \boldsymbol{\Lambda} & \mathbf{0} \\
\widetilde{\boldsymbol{K}} & \mathbf{0} & \mathbf{0} \\
\mathbf{0} & \boldsymbol{I} & \mathbf{0} \\
\mathbf{0} & \mathbf{0} & 1
\end{array}\right]
$$

where

$$
\begin{gathered}
\widetilde{\boldsymbol{K}}=\left[\begin{array}{cc}
\boldsymbol{K} & \mathbf{0} \\
\mathbf{0} & \boldsymbol{I} \\
-\boldsymbol{J}_{s} & -\boldsymbol{R}_{s}
\end{array}\right] \\
\boldsymbol{\Lambda}=\widetilde{\boldsymbol{K}}_{\ell}\left(\boldsymbol{I}-\boldsymbol{H}_{f}^{-1} \widetilde{\boldsymbol{J}}_{f}^{\top}\left(\widetilde{\boldsymbol{J}}_{f} \boldsymbol{H}_{f}^{-1} \widetilde{\boldsymbol{J}}_{f}^{\top}\right)^{-1} \widetilde{\boldsymbol{J}}_{f}\right)
\end{gathered}
$$

$$
\begin{gathered}
\widetilde{\boldsymbol{K}}_{\ell}=\left[\begin{array}{ccc}
\boldsymbol{K} & \mathbf{0} & \mathbf{0} \\
\mathbf{0} & \boldsymbol{I} & \mathbf{0}
\end{array}\right] \\
\boldsymbol{\Gamma}=\left[\frac{\partial \boldsymbol{\Lambda}}{\partial x_{s i}} \dot{\boldsymbol{x}}_{f}\right]
\end{gathered}
$$

$$
\begin{gathered}
\frac{\partial \boldsymbol{\Lambda}}{\partial x_{s i}}=\tilde{\boldsymbol{K}}_{\ell} \boldsymbol{H}_{f}^{-1} \frac{\partial \boldsymbol{H}_{f}}{\partial x_{f}} \boldsymbol{H}_{f}^{-1} \widetilde{\boldsymbol{J}}_{f}^{\top} \boldsymbol{\Omega} \widetilde{\boldsymbol{J}}_{f} \\
-\boldsymbol{H}_{f}^{-1} \frac{\partial \widetilde{\boldsymbol{J}}_{f}^{\top}}{\partial x_{f i}} \boldsymbol{\Omega} \widetilde{\boldsymbol{J}}_{f}-\boldsymbol{H}_{f}^{-1} \widetilde{\boldsymbol{J}}_{f}^{\top} \boldsymbol{\Omega} \frac{\partial \widetilde{\boldsymbol{J}}_{f}}{\partial x_{f_{i}}} \\
+\boldsymbol{H}_{f}^{-1} \widetilde{\boldsymbol{J}}_{f}^{\top} \boldsymbol{\Omega}\left(\frac{\partial \widetilde{\boldsymbol{J}}_{f}}{\partial x_{f}} \boldsymbol{H}_{f}^{-1} \widetilde{\boldsymbol{J}}_{f}^{\top}-\widetilde{\boldsymbol{J}}_{f} \boldsymbol{H}_{f}^{-1} \frac{\partial \boldsymbol{H}_{f}}{\partial x_{f i}} \boldsymbol{H}_{f}^{-1} \widetilde{\boldsymbol{J}}_{f}^{\top}\right. \\
\left.+\widetilde{\boldsymbol{J}}_{f} \boldsymbol{H}_{f}^{-1} \frac{\partial \widetilde{\boldsymbol{J}}_{f}^{\top}}{\partial x_{f}}\right) \boldsymbol{\Omega}_{\boldsymbol{J}_{f}} \tilde{\boldsymbol{K}}_{r} \\
\boldsymbol{\Omega}=\left(\widetilde{\boldsymbol{J}}_{f} \boldsymbol{H}_{f}^{-1} \widetilde{\boldsymbol{J}}_{f}^{\top}\right)^{-1} \\
\widetilde{\boldsymbol{K}}_{r}=\left[\begin{array}{cc}
\boldsymbol{K} & \mathbf{0} \\
\mathbf{0} & \boldsymbol{I} \\
\mathbf{0} & \mathbf{0}
\end{array}\right]
\end{gathered}
$$

The other partial derivatives are computed by using modified Newton-Eular formulations.

TABLE I

SPECIFICATIONS OF ROBOT.

\begin{tabular}{lrr}
\hline & length $[\mathrm{m}]$ & weight $[\mathrm{kg}]$ \\
\hline body & 0.6 & 20 \\
thigh & 0.3 & 10 \\
shin & 0.3 & 10 \\
\hline total height \& weight & 1.2 & 60 \\
\hline
\end{tabular}

TABLE II

CONTROL PARAMETERS.

\begin{tabular}{lr}
\hline stride $|\boldsymbol{S}|$ & $0.5[\mathrm{~m}]$ \\
period of one step $T$ & $0.5[\mathrm{~s}]$ \\
running speed $T /|\boldsymbol{S}|$ & $1[\mathrm{~m} / \mathrm{s}]$ \\
\hline
\end{tabular}

\section{Numerical Study of Five-Link Planar BiPed}

The proposed method is applied to a five-link planar biped robot. The specification is shown in Table I. The robot is 1.2 [m] height and $60[\mathrm{~kg}]$ weight. The coordinates are taken as shown in Fig. 1. The control parameters are selected as shown in Table II.

The optimal trajectories are computed as shown in Fig. 3Fig. 6. In Fig. 4, there are some discontinuous points due to the impact force at the instant of landing. Snapshots of the running motion are also shown in Fig. 7. Table III shows requirements for the actuators based on this result. It is found that very big power is required for knee joints. However, the total consumption power of them has small negative values. Therefore, the main work is done by the hip joints. Since the negative power is also big, the introduction of the 

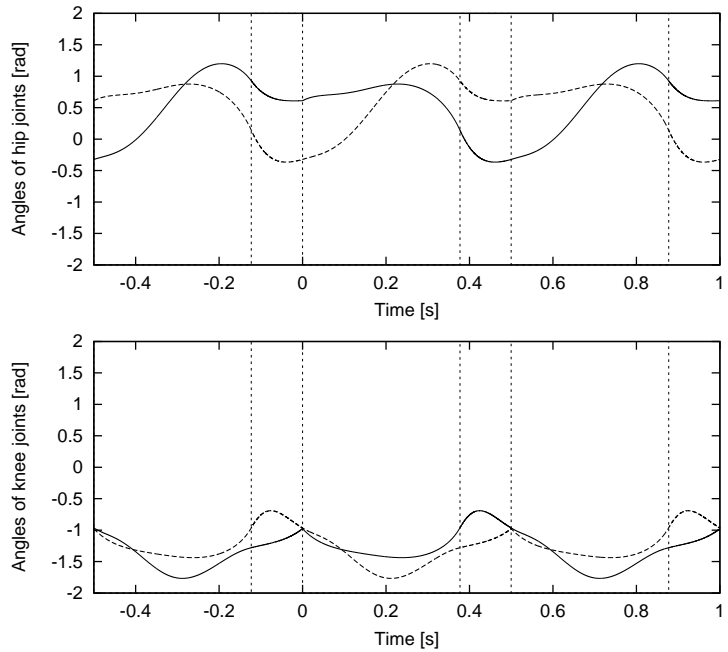

Fig. 3. Joint angles.
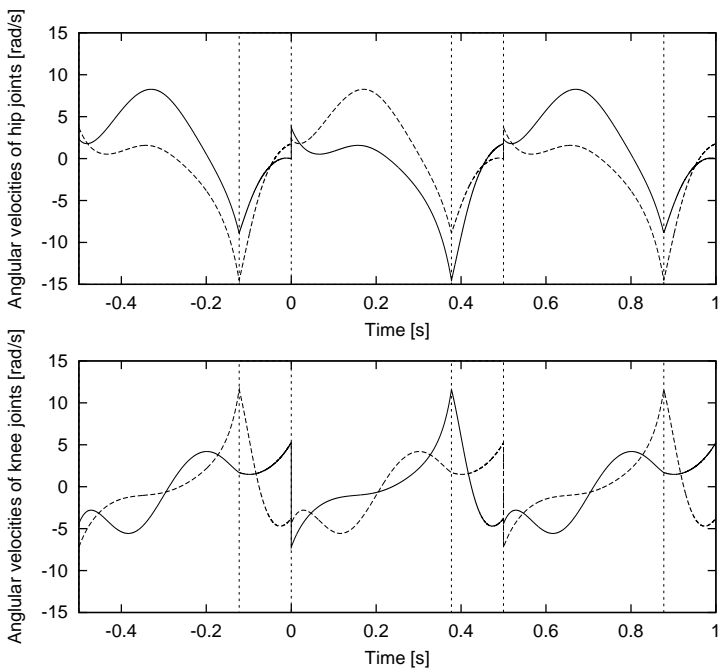

Fig. 4. Angular velocities of joints.

energy regeneration mechanism such as elastic actuators or combination of high backdrivable actuators and bidirectional power converters is effective to reduce the total consumption power.

\section{CONCLUSiON}

In this paper, the method to generate a trajectory of a running motion with minimum energy consumption is proposed. It is useful to know the lower bound of the consumption energy when we design the bipedal robot and select actuators. The exact and general formulation of optimal control for biped robots based on numerical representation of motion equation is proposed to solve exactly the minimum energy consumption trajectories. Through the numerical study of a five link planar biped robot, it is found that big peak power and torque is required for the knee joints but its consumption power is small and the main work is done by the hip joints.
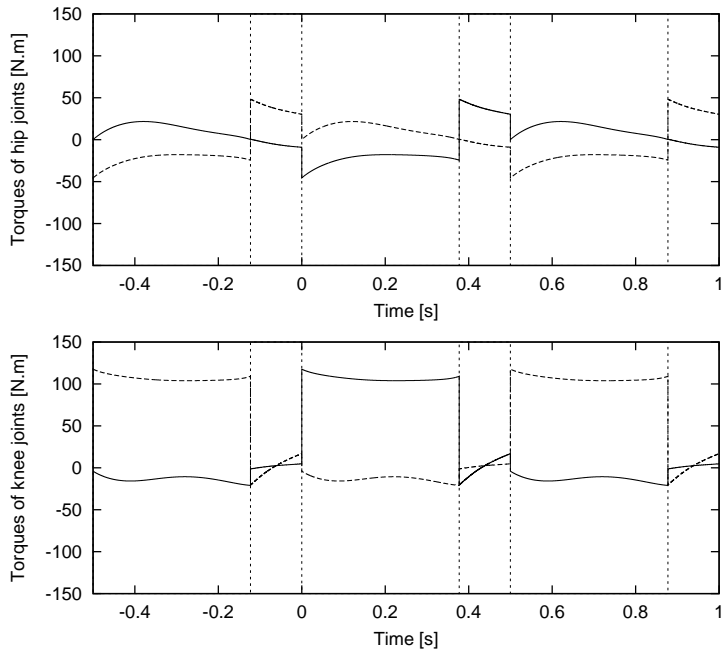

Fig. 5. Joint torques.
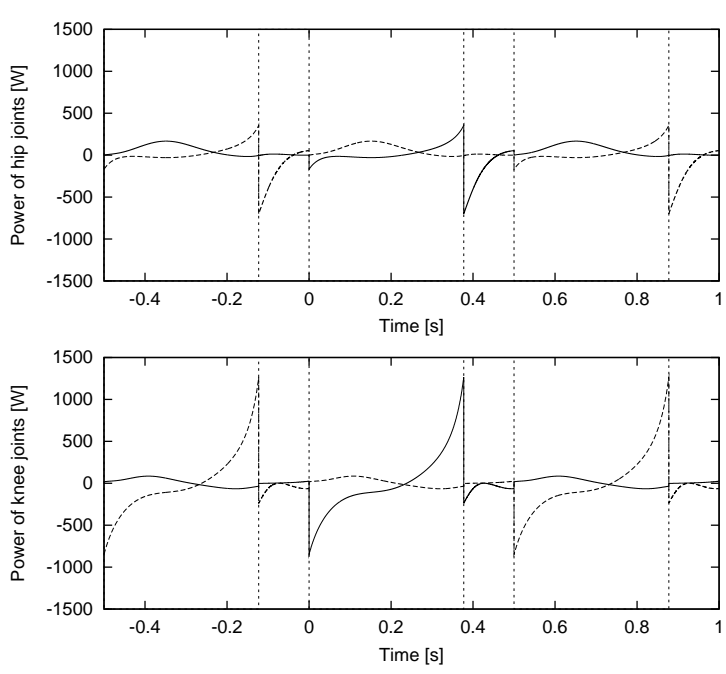

Fig. 6. Joint powers.

\section{APPENDIX}

At the instant of the landing, impact force $\delta \boldsymbol{f}$ is inflicted at the foot of support leg and the generalized velocity changes discontinuously. From (1), the generalized velocity after the collision is given by

$$
\dot{\boldsymbol{x}}_{+}=\dot{\boldsymbol{x}}_{-}+\boldsymbol{H}^{-1} \widetilde{\boldsymbol{J}}^{\top} \delta \boldsymbol{f}
$$

where $\dot{\boldsymbol{x}}_{+}$and $\dot{\boldsymbol{x}}_{-}$denote the generalized velocities after and before collision, respectively. $\widetilde{J}=[\boldsymbol{J}, \boldsymbol{R}, \boldsymbol{I}]$ is an extended Jacobian. Since it is support phase after the collision, the condition (3) holds, namely,

$$
\widetilde{\boldsymbol{J}} \dot{\boldsymbol{x}}_{+}=0 .
$$

Eliminating $\delta \boldsymbol{f}$ from above two equations, we have

$$
\dot{\boldsymbol{x}}_{+}=\left(\boldsymbol{I}-\boldsymbol{H}^{-1} \widetilde{\boldsymbol{J}}^{\top}\left(\widetilde{\boldsymbol{J}} \boldsymbol{H}^{-1} \widetilde{\boldsymbol{J}}^{\top}\right)^{-1} \widetilde{\boldsymbol{J}}\right) \dot{\boldsymbol{x}}_{-} .
$$




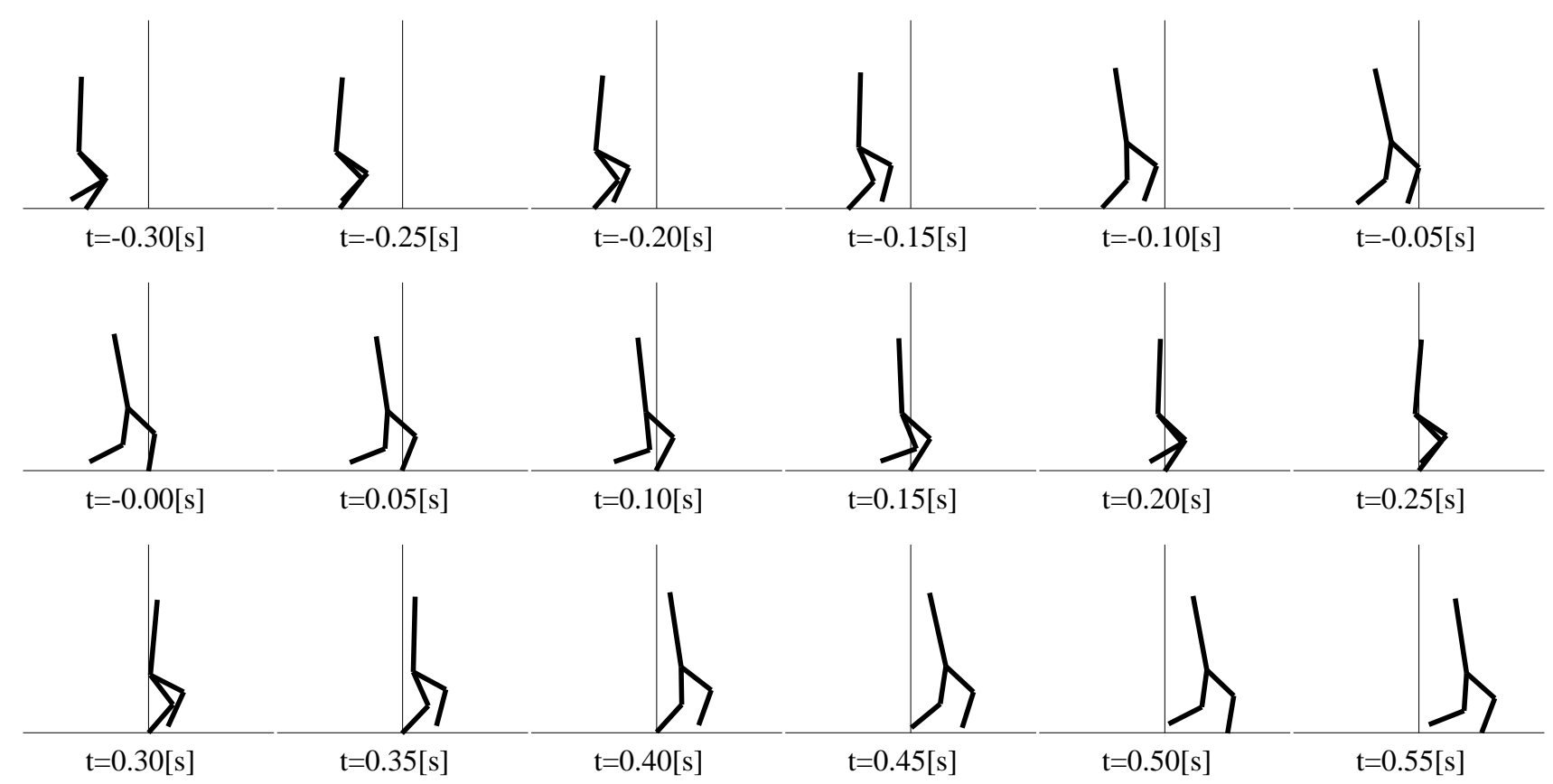

Fig. 7. Snapshots of running trajectory

TABLE III

ACTUATOR REQUIREMENTS.

\begin{tabular}{lrr}
\hline & hip & knee \\
\hline peak angular velocity [rad/s] & 14.5 & 11.6 \\
peak torque [N.m] & 48.2 & 117.6 \\
peak power (positive) [W] & 355 & 1265 \\
peak power (negative) [W] & -699 & -849 \\
\hline consumption power [W] & 27.9 & -5.37 \\
total consumption power of robot [W] & \multicolumn{2}{c}{45.1} \\
\hline
\end{tabular}

\section{REFERENCES}

[1] Miomir Vukobratović, Branislav Borovac, and Dragoljub Šurdilović, "Zero-Moment Point - Proper Interpretation and New Applications," Proc. Int. Conf. on Humanoids Robots, pp. 237-244, 2001.

[2] A. Goswami, "Foot-Rotation Indicator (FRI) Point: A New Gait Planning Tool to Evaluate Postural Stability of Biped Robot," Proc. IEEE ICRA, pp. 47-52, 1999.

[3] L. Roussel, C. Canudas-de-Wit, and A. Goswami, "Generation of Energy Optimal Complete Gait Cycles for Biped Robots," Proc. IEEE ICRA, pp. 2036-2041, 1998.

[4] J. Yamaguchi, E. Soga, S. Inoue, and A. Takanishi, "Development of a Bipedal Humanoid Robot - Control Method of Whole Body Cooperative Dynamic Biped Walking," Proc. IEEE ICRA, pp. 368-374, 1999.

[5] Yuusuke Sugahara, Tatsuro Endo, Hun-ok Lim, and Atsuo Takanishi, "Control and Experiments of a Multi-purpose Bipedal Locomotor with Parallel Mechanism," Proc. IEEE ICRA, pp. 4342-4347, 2003.
[6] Shuuji Kajita, Fujio Kanehiro, Kenji Kaneko, Kiyoshi Fujiwara, Kensuke Harada, Kazuhito Yokoi, and Hirohisa Hirukawa, "Biped Walking Pattern Generation by using Preview Control of Zero-Moment Point," Proc. IEEE ICRA, pp. 1620-1626, 2003.

[7] Kazuo Hirai, Masato Hirose, Yuji Haikawa, and Toru Takenaka, "The Development of Honda Humanoid Robot," Proc. IEEE ICRA, pp. 13211326, 1998.

[8] Koichi Nishiwaki, Satoshi Kagami, James J. Kuffner, Masayuki Inaba, Hirochika Inoue, "Online Humanoid Walking Control System and a Moving Goal Tracking Experiment," Proc. IEEE ICRA, pp. 911-916, 2003.

[9] K. Löffler, M. Gienger, F. Pfeiffer, "Sensor and Control Design of a Dynamically Stable Biped Robot," Proc. IEEE ICRA, pp. 484-490, 2003.

[10] Kemalettin Erbatur, Taro Takahashi, Akihiro Okazaki, Keisuke Obiya, and Atsuo Kawamura, "A study on the zero moment point measurement for biped walking robots," Proc. of IEEE Int. Workshop on Advanced Motion Control, pp. 431-436, 2002.

[11] Marc H. Raibert, Legged Robots That Balance, MIT Press, 1986.

[12] Jessica K. Hodgins, "Three-Dimensional Human Running," Proc. IEEE ICRA, pp. 3271-3277, 1996.

[13] Shuuji Kajita, Takashi Nagasaki, Kazuhito Yokoi, Kenji Kaneko, Kazuo Tanie, "Running Pattern Generation for a Humanoid Robot," Proc. IEEE ICRA, pp. 2755-2761, 2002.

[14] Yasutaka Fujimoto and Atsuo Kawamura, "Three Dimensional Digital Simulation and Autonomous Walking Control for Eight-axis Biped Robot," Proc. IEEE ICRA, pp. 2877-2884, 1995.

[15] Yasutaka Fujimoto and Atsuo Kawamura, "Simulation of an $\mathrm{Au}-$ tonomous Biped Walking Robot Including Environmental Force Interaction," IEEE Robotics and Automation Magazine, vol. 5, no. 2, pp. 33-42, 1998. 